KIAS-P98006

INFNCA-TH-9803

\title{
NEUTRINO SELF-ENERGY AND DISPERSION IN A MEDIUM WITH MAGNETIC FIELD
}

\author{
A. Erdas * \\ Dipartimento di Fisica dell' Università di Cagliari and I.N.F.N. Sezione di Cagliari, \\ Cittadella Universitaria, S.P. per Sestu Km 0.700, I-09042 Monserrato (CA), Italy \\ C. W. $\operatorname{Kim}^{\dagger}$ \\ Department of Physics and Astronomy, The Johns Hopkins University, Baltimore, MD $21218 \ddagger$ \\ School of Physics, Korea Institute for Advanced Study, Seoul 130-012, Korea \\ T. H. Lee ${ }^{\S}$ \\ Department of Physics, Soong Sil University, Seoul 156-743, Korea
}

\begin{abstract}
We calculate the one-loop thermal self-energy of a neutrino in a constant and homogeneous magnetic field to all orders in the magnetic field strength using Schwinger's proper time method. We obtain the dispersion relation under various conditions.
\end{abstract}

*erdas@vaxca2.unica.it

${ }^{\dagger}$ kim@eta.pha.jhu.edu

${ }^{\ddagger}$ permanent address

§thlee@physics.soongsil.ac.kr 


\section{INTRODUCTION}

The study of neutrino properties in a medium in the presence of a magnetic field is very important in the context of neutrino physics and of a possible solution to the solar neutrino puzzle, and it has been widely studied in the literature. It has been shown that, in the weak-field limit, the presence of a magnetic field in the medium modifies the neutrino index of refraction [1] , but, for massless left-handed neutrinos the interaction with the magnetic

field does neither flip chirality nor induce flavor transitions. In a more recent paper [2] the dispersion relation of neutrinos in magnetized media is investigated and the authors calculate the local part of the neutrino self-energy in a CP-asymmetric medium to all orders in the magnetic field strength $B$ and also the term linear in $B$ for the self energy in a CP-symmetric medium at temperatures much higher than the electron mass $m$ and much lower than the $W$-mass $M$. In this work we calculate the real part of the thermal self-energy in a medium

to all orders in the magnetic field, for all temperatures below the critical temperature of the $S U(2)_{L} \otimes U(1)$ standard model and for all momenta of the neutrino. Our more general result reproduces all the results in the literature [1, 2].

In Section II we calculate the thermal self-energy to all orders in $e B$ using the real-time method of finite temperature field theory. In Section III, after showing that our results for a CP-asymmetric plasma agree with those of Refs. [1],2], we derive a simple expression, valid to all orders in the magnetic field, for the self-energy and dispersion relation in the presence of a non-relativistic electron gas. In Section IV we use our main result of Section II to derive a simple expression for the self-energy in a CP-symmetric medium when $m \ll T \ll M$ to all orders in $e B$, and compute the dispersion relation. We conclude in Section V. 


\section{EXACT FINITE TEMPERATURE SELF-ENERGY IN A CONSTANT MAGNETIC FIELD}

In this Section we use the exact propagators in a constant, homogeneous magnetic field $\mathbf{B}$ to calculate the real part of the neutrino thermal self-energy in a magnetized medium. We take the magnetic field oriented along the $z$-direction and the metric to be $g^{\mu \nu}=\operatorname{diag}(-1,+1,+1,+1)$. Using Schwinger's proper time method [3], it is possible to

obtain the exact expression for the vacuum propagator of the charged lepton $S_{0}\left(x^{\prime}, x^{\prime \prime}\right)$ [3, 4 , the $W$-boson $G_{0}^{\mu \nu}\left(x^{\prime}, x^{\prime \prime}\right)$ [5] and the charged scalar $\Delta_{0}\left(x^{\prime}, x^{\prime \prime}\right)$ [4. 5 ]

$$
\begin{aligned}
& S_{0}\left(x^{\prime}, x^{\prime \prime}\right)= i \phi^{*}\left(x^{\prime}, x^{\prime \prime}\right) \int \frac{d^{4} k}{(2 \pi)^{4}} e^{i k \cdot\left(x^{\prime}-x^{\prime \prime}\right)} \int_{0}^{\infty} \frac{d s}{\cos e B s} \exp \left[-i s\left(m^{2}-i \epsilon+k_{\|}^{2}+k_{\perp}^{2} \frac{\tan e B s}{e B s}\right)\right] \\
& \times {\left[\left(m-\not k_{\|}\right) e^{-i e B s \sigma_{3}}-\frac{\not k_{\perp}}{\cos e B s}\right] } \\
& G_{0}^{\mu \nu}\left(x^{\prime}, x^{\prime \prime}\right)= i \phi\left(x^{\prime}, x^{\prime \prime}\right) \int \frac{d^{4} k}{(2 \pi)^{4}} e^{i k \cdot\left(x^{\prime}-x^{\prime \prime}\right)} \int_{0}^{\infty} \frac{d s}{\cos e B s} \exp \left[-i s\left(k_{\|}^{2}+k_{\perp}^{2} \frac{\tan e B s}{e B s}\right)\right] \\
& \times\left\{e^{-i s\left(M^{2}-i \epsilon\right)}\left[g_{\|}^{\mu \nu}+\left(e^{2 e F s}\right)_{\perp}^{\mu \nu}\right]+\left(\frac{e^{-i s\left(M^{2}-i \epsilon\right)}-e^{-i s\left(\xi M^{2}-i \epsilon\right)}}{M^{2}}\right)\right. \\
& \times {\left[\left(k^{\mu}+k_{\lambda} F^{\mu \lambda} \frac{\tan e B s}{B}\right)\left(k^{\nu}+k_{\rho} F^{\rho \nu} \frac{\tan e B s}{B}\right)\right.} \\
&\left.\left.+i \frac{e}{2}\left(F^{\mu \nu}-g_{\perp}^{\mu \nu} B \tan e B s\right)\right]\right\} \\
& \Delta_{0}\left(x^{\prime}, x^{\prime \prime}\right)=i \phi\left(x^{\prime}, x^{\prime \prime}\right) \int \frac{d^{4} k}{(2 \pi)^{4}} e^{i k \cdot\left(x^{\prime}-x^{\prime \prime}\right)} \int_{0}^{\infty} \frac{d s}{\cos e B s} \exp \left[-i s\left(\xi M^{2}-i \epsilon+k_{\|}^{2}+k_{\perp}^{2} \frac{\tan e B s}{e B s}\right)\right],
\end{aligned}
$$

where the subscript 0 on the propagators indicates explicitly that these are vacuum propagators, $-e$ is the charge of the electron, $m$ the electron mass, $M$ the $W$-mass, $\xi$ the gauge parameter, $B=|\mathbf{B}|, F^{\mu \nu}$ is the electromagnetic field strength tensor whose only non-vanishing components are $F^{12}=B=-F^{21}$. Also in Eqs. (2.1), (2.2), and (2.3) we have

$$
\phi\left(x^{\prime}, x^{\prime \prime}\right)=\exp \left(i \frac{e}{2} x_{\mu}^{\prime \prime} F^{\mu \nu} x_{\nu}^{\prime}\right),
$$




$$
\sigma_{3}=\sigma^{12}=\frac{i}{2}\left[\gamma^{1}, \gamma^{2}\right]
$$

for any 4 -vector $a^{\mu}$ we define $a_{\|}^{\mu}$ and $a_{\perp}^{\mu}$ as

$$
a_{\|}^{\mu}=\left(a^{0}, 0,0, a^{3}\right) \quad, \quad a_{\perp}^{\mu}=\left(0, a^{1}, a^{2}, 0\right)
$$

and we have

$$
\left(e^{2 e F s}\right)_{\perp}^{\mu \nu}=\left(g^{\mu \nu}\right)_{\perp} \cos 2 e B s+F^{\mu \nu} \frac{\sin 2 e B s}{B} .
$$

This notation follows closely the one in Refs. [4,5]. The real-time thermal propagators are easily constructed starting from the proper-time form of the propagators in vacuum. For the fermion we write the vacuum propagator as

$$
S_{0}\left(x^{\prime}, x^{\prime \prime}\right)=\phi^{*}\left(x^{\prime}, x^{\prime \prime}\right) \int \frac{d^{4} k}{(2 \pi)^{4}} e^{i k \cdot\left(x^{\prime}-x^{\prime \prime}\right)} S_{0}(k),
$$

and obtain the following expression [2,6] of the propagator $S\left(x^{\prime}, x^{\prime \prime}\right)$ in the presence of an external magnetic field and a plasma at non-zero temperature and density,

$$
S\left(x^{\prime}, x^{\prime \prime}\right)=\phi^{*}\left(x^{\prime}, x^{\prime \prime}\right) \int \frac{d^{4} k}{(2 \pi)^{4}} e^{i k \cdot\left(x^{\prime}-x^{\prime \prime}\right)} S(k),
$$

where the translationally invariant part $S(k)$ of the propagator in a medium is defined in terms of the translationally invariant part $S_{0}(k)$ of the vacuum propagator and of the fermion occupation number $f_{F}\left(k^{0}\right)$ at temperature $T$ and chemical potential $\mu$

$$
S(k)=S_{0}(k)-f_{F}\left(k^{0}\right)\left[S_{0}(k)-S_{0}^{*}(k)\right] .
$$

Notice that in Eq. (2.10) the piece proportional to the occupation number represents the thermal part of the propagator, which we call $S_{T}(k)$. The fermion occupation number is defined as

$$
f_{F}\left(k^{0}\right)=f_{F}^{+}\left(k^{0}\right) \theta\left(k^{0}\right)+f_{F}^{-}\left(k^{0}\right) \theta\left(-k^{0}\right)
$$

with 


$$
f_{F}^{ \pm}\left(k^{0}\right)=\frac{1}{e^{ \pm\left(k^{0}-\mu\right) / T}+1}
$$

The same procedure is employed to obtain the propagators in a medium of the $W$-boson, $G^{\mu \nu}\left(x^{\prime}, x^{\prime \prime}\right)$, and of the charged scalar, $\Delta\left(x^{\prime}, x^{\prime \prime}\right)$ :

$$
G^{\mu \nu}\left(x^{\prime}, x^{\prime \prime}\right)=\phi\left(x^{\prime}, x^{\prime \prime}\right) \int \frac{d^{4} k}{(2 \pi)^{4}} e^{i k \cdot\left(x^{\prime}-x^{\prime \prime}\right)} G^{\mu \nu}(k)
$$

with

$$
G^{\mu \nu}(k)=G_{0}^{\mu \nu}(k)+f_{B}\left(k^{0}\right)\left[G_{0}^{\mu \nu}(k)-G_{0}^{\mu \nu *}(k)\right]
$$

where the boson occupation number $f_{B}\left(k^{0}\right)$ is defined as

$$
f_{B}\left(k^{0}\right)=\frac{1}{e^{\left|k^{0}\right| / T}-1}
$$

and

$$
\Delta\left(x^{\prime}, x^{\prime \prime}\right)=\phi\left(x^{\prime}, x^{\prime \prime}\right) \int \frac{d^{4} k}{(2 \pi)^{4}} e^{i k \cdot\left(x^{\prime}-x^{\prime \prime}\right)} \Delta(k)
$$

with

$$
\Delta(k)=\Delta_{0}(k)+f_{B}\left(k^{0}\right)\left[\Delta_{0}(k)-\Delta_{0}^{*}(k)\right]
$$

The three Feynman diagrams shown in Fig. 1 contribute to the neutrino self-energy in a medium in magnetic field. The tadpole diagram $\Sigma_{t a d}$ gives only a local contribution to the self-energy, while the bubble diagram with a $W$-boson $\Sigma_{b u b}\left(x^{\prime}, x^{\prime \prime}\right)$ and the bubble diagram with a scalar $\Sigma_{\text {scal }}\left(x^{\prime}, x^{\prime \prime}\right)$ contain also non-local terms. They are given by

$$
\begin{gathered}
\Sigma_{t a d}=-i g_{Z}^{2} \operatorname{tr}\left[\gamma_{\mu}\left(c_{V}^{f}-c_{A}^{f} \gamma_{5}\right) S(x, x)\right] \gamma_{\nu} \gamma_{L} Z^{\mu \nu}(0), \\
\Sigma_{b u b}\left(x^{\prime}, x^{\prime \prime}\right)=i\left(\frac{g}{\sqrt{2}}\right)^{2} \gamma_{R} \gamma_{\mu} S\left(x^{\prime}, x^{\prime \prime}\right) \gamma_{\nu} \gamma_{L} G^{\mu \nu}\left(x^{\prime}, x^{\prime \prime}\right), \\
\Sigma_{s c a l}\left(x^{\prime}, x^{\prime \prime}\right)=i\left(\frac{g}{\sqrt{2}}\right)^{2}\left(\frac{m}{M}\right)^{2} \gamma_{R} S\left(x^{\prime}, x^{\prime \prime}\right) \gamma_{L} \Delta\left(x^{\prime}, x^{\prime \prime}\right),
\end{gathered}
$$


where $Z^{\mu \nu}(0)$ is the vacuum propagator of the neutral $Z$-boson at zero momentum,

$$
g_{Z}=\frac{g}{2 \cos \theta_{W}}, \gamma_{R}=\frac{1+\gamma_{5}}{2}, \gamma_{L}=\frac{1-\gamma_{5}}{2}
$$

and the index $f$ runs over $e, p, n$, since we consider a medium where electrons, protons and neutrons are present, and

$$
c_{V}^{e}=-c_{V}^{p}=c_{V}, \quad c_{A}^{e}=-c_{A}^{p}=c_{A}, \quad c_{V}^{n}=c_{A}^{n}=-\frac{1}{2}
$$

with

$$
c_{V}=-\frac{1}{2}+2 \sin ^{2} \theta_{W}, \quad c_{A}=-\frac{1}{2}
$$

We compute the self-energy of an electron neutrino in a medium. (A generalization to muon or tau neutrino is straightforward.) Using the expression of the fermion propagator from Eqs. (2.1), (2.9), and (2.10) we obtain the following expression of the tadpole term in a medium with non-vanishing electron chemical potential

$$
\begin{aligned}
\Sigma_{t a d}= & 4 \frac{g_{Z}^{2}}{M_{Z}^{2}} \int_{-\infty}^{+\infty} \frac{d k}{(2 \pi)^{4}} k f_{F}(k) \int_{-\infty}^{+\infty} d s e^{-\left[i s\left(m^{2}-k^{2}\right)+|s| \epsilon\right]}\left(\frac{\pi}{i s}\right)^{3 / 2}(e B s) \\
& \times \gamma_{R}\left[c_{V} \gamma^{0} \cot e B s-i c_{A} \gamma^{3}\right]
\end{aligned}
$$

where the term linear in $B$ has a negative sign because in this paper we take the electron charge to be $-e$. The generalization to the case of non-zero nucleon chemical potential is quite straightforward and will be discussed briefly in Sec. III. After we change variable in the $k$-integration and perform the $s$-integration, Eq. (2.24) becomes [2]

$$
\Sigma_{t a d}=\frac{g_{Z}^{2}}{M_{Z}^{2}} \gamma_{R}\left[c_{V} \gamma^{0}\left(N_{e}-N_{\bar{e}}\right)-c_{A} \gamma^{3}\left(N_{e}^{0}-N_{\bar{e}}^{0}\right)\right] \gamma_{L}
$$

where the net number density of electrons in a magnetic field is defined as [2]

$$
N_{e}-N_{\bar{e}}=\frac{|e B|}{2 \pi^{2}} \int_{0}^{\infty} d k_{z} \sum_{n=0}^{\infty} \sum_{\lambda= \pm 1}\left[f_{F}^{+}\left(E_{n, \lambda, k_{z}}\right)-f_{F}^{-}\left(-E_{n, \lambda, k_{z}}\right)\right]
$$

and the energies of the Landau levels are

$$
E_{n, \lambda, k_{z}}^{2}=m^{2}+k_{z}^{2}+|e B|(2 n+1-\lambda)
$$


where $n$ is the orbital quantum number and $\lambda$ the spin quantum number. The quantity $N_{e}^{0}-N_{\bar{e}}^{0}$ in Eq. (2.25) represents the net number density in the lowest Landau level, and is given by

$$
N_{e}^{0}-N_{\bar{e}}^{0}=\frac{|e B|}{2 \pi^{2}} \int_{0}^{\infty} d k_{z}\left[f_{F}^{+}\left(E_{0,1, k_{z}}\right)-f_{F}^{-}\left(-E_{0,1, k_{z}}\right)\right]
$$

Starting from Eqs. (2.19) and (2.20) we obtain the expressions of the two bubble contributions to the self-energy, using the boson and scalar propagators of Eqs. (2.2)-(2.3) and (2.13)-(2.17). We do the calculation in the Feynman gauge, and take $\xi=1$. The two particles in the loop are oppositely charged, and therefore the phase factors $\phi\left(x^{\prime}, x^{\prime \prime}\right)$ cancel, yielding a result which is explicitly translationally invariant

$$
\Sigma_{b u b}\left(x^{\prime}, x^{\prime \prime}\right)=\int \frac{d^{4} p}{(2 \pi)^{4}} e^{i p \cdot\left(x^{\prime}-x^{\prime \prime}\right)} \Sigma_{b u b}(p) .
$$

The bubble contribution to the self-energy contains a vacuum part and two thermal parts due to contributions of thermal fermions, which in this case are electrons, and thermal bosons respectively

$$
\Sigma_{b u b}(p)=\Sigma_{b u b}^{0}(p)+\Sigma_{b u b}^{F}(p)+\Sigma_{b u b}^{B}(p)
$$

The scalar contribution $\Sigma_{\text {scal }}$ is also translationally invariant and contains three pieces as in Eq. (2.30). For the expression of the vacuum part of $\Sigma_{b u b}$ we refer the reader to Ref. [5], here we are interested in the thermal parts which, in the $\xi=1$ gauge, are given by

$$
\begin{aligned}
\Sigma_{b u b}^{F}(p)= & i \frac{g^{2}}{2} \gamma_{R} \gamma_{\mu} \int \frac{d^{4} k}{(2 \pi)^{4}} f_{F}\left(k^{0}\right) \int_{-\infty}^{+\infty} d s \frac{e^{-\left[i s\left(m^{2}+\tilde{k}^{2}\right)+|s| \epsilon\right]}}{\cos e B s}\left[\left(m-\not k_{\|}\right) e^{-i e B s \sigma_{3}}-\frac{\not k_{\perp}}{\cos e B s}\right] \\
& \times \int_{0}^{\infty} d t \frac{e^{-i t\left(M^{2}+\tilde{q}^{2}-i \epsilon\right)}}{\cos e B t}\left[g_{\|}^{\mu \nu}+\left(e^{2 e F t}\right)_{\perp}^{\mu \nu}\right] \gamma_{\nu} \gamma_{L}, \\
\Sigma_{b u b}^{B}(p)= & -i \frac{g^{2}}{2} \gamma_{R} \gamma_{\mu} \int \frac{d^{4} k}{(2 \pi)^{4}} f_{B}\left(k^{0}\right) \int_{0}^{\infty} d s \frac{e^{-i s\left(m^{2}+\tilde{q}^{2}-i \epsilon\right)}}{\cos e B s}\left[\left(m-\not q_{\|}\right) e^{-i e B s \sigma_{3}}-\frac{\not q_{\perp}}{\cos e B s}\right] \\
& \times \int_{-\infty}^{+\infty} d t \frac{e^{-\left[i t\left(M^{2}+\tilde{k}^{2}\right)+|t| \epsilon\right]}}{\cos e B t}\left[g_{\|}^{\mu \nu}+\left(e^{2 e F t}\right)_{\perp}^{\mu \nu}\right] \gamma_{\nu} \gamma_{L},
\end{aligned}
$$

where $q=p-k$, and we use the shortened notation 


$$
s \tilde{k}^{2}=s k_{\|}^{2}+k_{\perp}^{2} \frac{\tan e B s}{e B}, \quad t \tilde{k}^{2}=t k_{\|}^{2}+k_{\perp}^{2} \frac{\tan e B t}{e B},
$$

and the same for $\tilde{q}$. The $\vec{k}$-integration is done after a suitable change of the $\vec{k}$-variable, to obtain

$$
\begin{aligned}
\Sigma_{b u b}^{F}(p)= & -\frac{g^{2}}{2} \int_{-\infty}^{+\infty} \frac{d k^{0}}{(2 \pi)^{3}} f_{F}\left(k^{0}\right) \int_{0}^{\infty} d s \int_{-1}^{+1} d u\left(\frac{\pi}{i s}\right)^{1 / 2} \frac{e B s}{\sin e B s} \exp \left\{-i s\left[u m^{2}+(1-u) M^{2}\right.\right. \\
& \left.\left.-u\left(k^{0}\right)^{2}-(1-u)\left(p^{0}-k^{0}\right)^{2}+u(1-u)\left(p^{3}\right)^{2}+\frac{\sin e B s u \sin e B s(1-u)}{e B s \sin e B s} p_{\perp}^{2}\right]\right\} \\
& \times \gamma_{R}\left[e^{-i \sigma_{3} e B s(2-u)}\left(\gamma^{0} k_{0}+(1-u) \gamma^{3} p_{3}\right)+\frac{\sin e B s(1-u)}{\sin e B s} \not p_{\perp}\right] \gamma_{L}, \\
\Sigma_{b u b}^{B}(p)= & \frac{g^{2}}{2} \int_{-\infty}^{+\infty} \frac{d k^{0}}{(2 \pi)^{3}} f_{B}\left(k^{0}\right) \int_{0}^{\infty} d s \int_{-1}^{+1} d u\left(\frac{\pi}{i s}\right)^{1 / 2} \frac{e B s}{\sin e B s} \exp \left\{-i s\left[(1-u) m^{2}+u M^{2}\right.\right. \\
& \left.\left.-u\left(k^{0}\right)^{2}-(1-u)\left(p^{0}-k^{0}\right)^{2}+u(1-u)\left(p^{3}\right)^{2}+\frac{\sin e B s u \sin e B s(1-u)}{e B s \sin e B s} p_{\perp}^{2}\right]\right\} \\
& \times \gamma_{R}\left[e^{-i \sigma_{3} e B s(1+u)}\left(\gamma^{0} p_{0}-\gamma^{0} k_{0}+u \gamma^{3} p_{3}\right)+\frac{\sin e B s u}{\sin e B s} \not p_{\perp}\right] \gamma_{L} .
\end{aligned}
$$

Notice also that we have changed the variables of the $s$ - and $t$-integrations in the following way: $s \rightarrow s u, t \rightarrow s(1-u)$ in the fermionic part and $s \rightarrow s(1-u), t \rightarrow s u$ in the bosonic part.

The scalar contributions $\Sigma_{\text {scal }}^{F}$ and $\Sigma_{\text {scal }}^{B}$ are easily obtained by replacing $g^{2}$ with $\frac{g^{2} m^{2}}{2 M^{2}}$ in Eqs. (2.34), (2.35) and $e^{-i \sigma_{3} e B s(2-u)}$ with $e^{-i \sigma_{3} e B s u}$ in Eq. (2.34) and $e^{-i \sigma_{3} e B s(1+u)}$ with $e^{-i \sigma_{3} e B s(1-u)}$ in Eq. (2.35), respectively.

The expressions in Eqs. (2.34) and (2.35) are some of the main results of our paper, which give the exact non-local thermal self-energy for all values of the magnetic field, all values of the temperature that are below the critical temperature of $250 \mathrm{GeV}$, all values of electron chemical potential, and all values of the neutrino 4-momentum.

\section{III. $C P$-ASYMMETRIC MEDIUM}

We consider now a $C P$-asymmetric medium at temperature $T \ll M$ and a magnetic field $B \ll M^{2} / e$. Under these conditions we can neglect thermal corrections to the $W$-propagator and simply use the vacuum propagator in the contact approximation 


$$
G^{\mu \nu}\left(x^{\prime}, x^{\prime \prime}\right) \simeq G_{0}^{\mu \nu}\left(x^{\prime}, x^{\prime \prime}\right) \simeq \phi\left(x^{\prime}, x^{\prime \prime}\right) \int \frac{d^{4} k}{(2 \pi)^{4}} e^{i k \cdot\left(x^{\prime}-x^{\prime \prime}\right)} \frac{g^{\mu \nu}}{M^{2}}
$$

To leading order in $1 / M^{2}$ the scalar contribution is also negligible.

Using the Fierz-type identity [1]

$$
\gamma_{R} \gamma^{\mu} A \gamma_{\mu} \gamma_{L}=-\gamma_{R} \gamma^{\mu} \operatorname{Tr}\left[\gamma_{\mu} \gamma_{L} A\right]
$$

it is evident that $\Sigma_{b u b}^{F}$ in the contact approximation is obtained from $\Sigma_{t a d}$ of Eq. (2.18) by making the following replacements

$$
\frac{g_{Z}^{2}}{M_{Z}^{2}} \rightarrow \frac{g^{2}}{2 M^{2}}, c_{V} \rightarrow \frac{1}{2}, c_{A} \rightarrow \frac{1}{2}
$$

Let us consider the thermal self-energy of an electron neutrino in an electron-rich medium, to leading order in $1 / M^{2}$. The sum of the tadpole and bubble diagrams in the contact approximation is readily obtained making use of Eq.(2.24) and of the replacements mentioned in Eq. (3.3)

$$
\begin{aligned}
\Sigma= & \frac{g^{2}}{M^{2}} \int_{-\infty}^{+\infty} \frac{d k}{(2 \pi)^{4}} k f_{F}(k) \int_{-\infty}^{+\infty} d s e^{-\left[i s\left(m^{2}-k^{2}\right)+|s| \epsilon\right]}\left(\frac{\pi}{i s}\right)^{3 / 2}(e B s) \\
& \times \gamma_{R}\left[\left(1+c_{V}\right) \gamma^{0} \cot e B s-i\left(1+c_{A}\right) \gamma^{3}\right] \gamma_{L},
\end{aligned}
$$

where we have also used $M_{Z}=M / \cos \theta_{W}$.

In a weak-field limit we can write the self-energy as

$$
\Sigma=\frac{g^{2}}{4 M^{2}} \gamma_{R}\left[\left(1+c_{V}\right) \gamma^{0}\left(n_{e}-n_{\bar{e}}\right)-\left(1+c_{A}\right) \gamma^{3}\left(N_{e}^{0}-N_{\bar{e}}^{0}\right)\right] \gamma_{L}+O\left(B^{2}\right)
$$

where the net number density of electrons in the absence of a magnetic field is

$$
n_{e}-n_{\bar{e}}=2 \int \frac{d^{3} k}{(2 \pi)^{3}}\left[f_{F}^{+}\left(\omega_{k}\right)-f_{F}^{-}\left(-\omega_{k}\right)\right]
$$

with $\omega_{k}=\sqrt{|\vec{k}|^{2}+m^{2}}$, and $N_{e}^{0}-N_{\bar{e}}^{0}$ is the net number density of electrons in the lowest Landau level given by Eq. (2.28). The quantity $\gamma^{3}\left(N_{e}^{0}-N_{\bar{e}}^{0}\right)$ can be written as

$$
-2 e \not P \int \frac{d^{3} k}{(2 \pi)^{3}} \frac{1}{2 \omega_{k}} \frac{d}{d \omega_{k}}\left[f_{F}^{+}\left(\omega_{k}\right)-f_{F}^{-}\left(-\omega_{k}\right)\right]
$$


and therefore our results for the weak-field limit are in agreement with those found in Ref. [1]. Note that the authors of Ref. [1] use the conventions opposite to ours $g^{\mu \nu}=$ $\operatorname{diag}(+1,-1,-1,-1)$ and $e<0$ for the electron.

Let us now consider a nonrelativistic and nondegenerate limit of the electron gas. Using the expansion of the cotangent as a power series involving the Bernoulli numbers $A_{l}$, we rewrite Eq. (3.4) as

$$
\begin{aligned}
\Sigma= & \frac{g^{2}}{4 M^{2}} \gamma_{R}\left\{\left(1+c_{V}\right) \gamma^{0}\left[1-\sum_{l=1}^{\infty} \frac{(-1)^{l}}{(2 l) !}\left|A_{2 l}\right|(2 e B)^{2 l}\left(\frac{\partial}{\partial m^{2}}\right)^{2 l}\right]+\left(1+c_{A}\right) e \not B \frac{\partial}{\partial m^{2}}\right\} \gamma_{L} \\
& \times 2 \int \frac{d^{3} k}{(2 \pi)^{3}}\left[f_{F}^{+}\left(\omega_{k}\right)-f_{F}^{-}\left(-\omega_{k}\right)\right] .
\end{aligned}
$$

For a nonrelativistic and nondegenerate electron gas [1]

$$
f_{F}^{+} \simeq e^{-\beta\left(\omega_{k}-\mu\right)} \text { and } f_{F}^{-}=0
$$

with the electron energy $\omega_{k} \simeq m+|\vec{k}|^{2} / 2 m$, and therefore

$$
\frac{\partial f_{F}^{+}}{\partial m^{2}} \simeq-\frac{\beta}{2 m} f_{F}^{+}
$$

So for the nonrelativistic electron gas Eq. (3.7) yields

$$
\Sigma=\gamma_{R}(b \not u+c \not B) \gamma_{L}
$$

with

$$
b=-\frac{g^{2}}{4 M^{2}}\left(1+c_{V}\right) n_{e} \frac{\mu_{B} B}{T} \operatorname{coth}\left(\frac{\mu_{B} B}{T}\right) \quad \text { and } \quad c=-\frac{g^{2}}{4 M^{2}}\left(1+c_{A}\right) n_{e} \frac{\mu_{B}}{T}
$$

where $\mu_{B}=e / 2 m$ is the Bohr magneton, $n_{e}$ is the number density of electrons as defined in Eq. (3.6), $u^{\mu}$ is the 4 -velocity of the medium and $\not \mu=-\gamma^{0}$ in the reference frame of the medium. This result is exact to all orders in the magnetic field provided that $T<<m$ and $\mu_{B} B<\pi T$. The dispersion relation for electron neutrino in a nonrelativistic electron gas is obtained by setting $(\not p+b \not h+c \not B)^{2}=0$, which, to lowest order in $1 / M^{2}$, yields

$$
E=|\vec{p}|+c \hat{p} \cdot \vec{B}-b
$$


where the neutrino 4-momentum is $p^{\mu}=(E, \vec{p})$. The dispersion relation of Eq. (3.10) shows that electron neutrinos in the presence of a magnetic field and in a nonrelativistic electron gas have an anisotropic index of refraction $n$

$$
n=1+\frac{g^{2}}{4 M^{2}} n_{e}\left(\frac{\mu_{B} B}{T}\right) \frac{\left(1+c_{A}\right) \hat{p} \cdot \hat{B}-\left(1+c_{V}\right) \operatorname{coth}\left(\frac{\mu_{B} B}{T}\right)}{|\vec{p}|}
$$

and an effective mass $m_{\nu_{e}}=-b$. This $m_{\nu_{e}}$ is calculated from the one-loop diagram at finite temperature in a magnetic field and is exact to all orders in the magnetic field strength for $\mu_{B} B<\pi T$. This mass is similar in magnitude in the limit $\mu_{B} B / T \rightarrow 0$ as the well-known effective potential $V_{C}=\sqrt{2} G_{F} n_{e}$ induced by the coherent scatterings of a electron neutrino off electrons [7].

Finally we briefly consider the self-energy of an electron neutrino in a medium where electrons, neutrons and protons are all present. Our results in the contact approximation agree with those of Ref. [2], and we write the self-energy exact to all orders in $B$ as

$$
\begin{aligned}
\Sigma= & \frac{g^{2}}{4 M^{2}} \gamma_{R}\left\{\left[\left(1+c_{V}\right)\left(N_{e}-N_{\bar{e}}\right)-c_{V}\left(N_{p}-N_{\bar{p}}\right)-\frac{1}{2}\left(N_{n}-N_{\bar{n}}\right)\right] \gamma^{0}\right. \\
& \left.-\left(1+c_{A}\right)\left(N_{e}^{0}-N_{\bar{e}}^{0}\right) \gamma^{3}\right\} \gamma_{L},
\end{aligned}
$$

where $N_{f}-N_{\bar{f}}$ is the net number density of fermions in a magnetic field as defined in Eq. (2.26) and $N_{e}^{0}-N_{\bar{e}}^{0}$ is the net number density of electrons in the lowest Landau level, as defined in Eq. (2.28). In this Section we are considering temperatures much smaller than the typical nucleon mass and fields that are not extremely strong, therefore the nucleon magnetization is suppressed by their heavier mass relative to the electrons [2]. For this reason we do not have a term proportional to $\gamma^{3}$ times the nucleon density in Eq. (3.12).

The dispersion relation for an electron neutrino is obtained immediately [2]

$$
E=-b+|\vec{p}+\vec{c}|
$$

with

$$
b=-\frac{g^{2}}{4 M^{2}}\left[\left(1+c_{V}\right)\left(N_{e}-N_{\bar{e}}\right)-c_{V}\left(N_{p}-N_{\bar{p}}\right)-\frac{1}{2}\left(N_{n}-N_{\bar{n}}\right)\right]
$$


and

$$
\vec{c}=-\frac{g^{2}}{4 M^{2}}\left(1+c_{A}\right)\left(N_{e}^{0}-N_{\bar{e}}^{0}\right) \hat{B}
$$

\section{IV. $C P$-SYMMETRIC MEDIUM}

In a medium that is charge-symmetric, the local contributions to the self-energy vanish, and we only need to consider the two bubble diagrams, but only the one with $W$-boson is significant because the scalar diagram is suppressed by a factor of $m^{2} / M^{2}$.

In the weak-field approximation and in the $\xi=1$ gauge, we obtain the following expression for the thermal part of the neutrino self-energy, valid for any temperature below the critical temperature of the $S U(2)_{L} \otimes U(1)$ model, and for all values of the neutrino 4-momentum

$$
\begin{aligned}
\Sigma^{F}= & \frac{g^{2}}{16 \pi^{2}} \int_{0}^{\infty} k^{2} d k\left(\frac{\partial}{\partial m^{2}}+2 \frac{\partial}{\partial M^{2}}\right) f_{F}\left(\omega_{k}\right) \gamma_{R}\left\{\frac{e \not p}{k|\vec{p}|} L_{1}^{-}(k)\right. \\
& \left.+\frac{e \gamma^{0} \vec{B} \cdot \vec{p}}{\omega_{k}|\vec{p}|^{2}}\left[4-\frac{M^{2}-m^{2}-E^{2}+|\vec{p}|^{2}}{2 k|\vec{p}|} L_{1}^{+}(k)-\frac{E \omega_{k}}{k|\vec{p}|} L_{1}^{-}(k)\right]\right\} \gamma_{L}, \\
\Sigma^{B}= & \frac{g^{2}}{16 \pi^{2}} \int_{0}^{\infty} k^{2} d k\left(\frac{\partial}{\partial m^{2}}+2 \frac{\partial}{\partial M^{2}}\right) f_{B}\left(\Omega_{k}\right) \gamma_{R}\left\{\frac{e B \sigma_{3} \not p_{\|}}{\Omega_{k} k|\vec{p}|} L_{2}^{+}(k)+\frac{e \not B}{k|\vec{p}|} L_{2}^{-}(k)\right. \\
& \left.+\frac{e \gamma^{0} \vec{B} \cdot \vec{p}}{\Omega_{k}|\vec{p}|^{2}}\left[4+\frac{m^{2}-M^{2}-E^{2}+|\vec{p}|^{2}}{2 k|\vec{p}|} L_{2}^{+}(k)-\frac{E \Omega_{k}}{k|\vec{p}|} L_{2}^{-}(k)\right]\right\} \gamma_{L},
\end{aligned}
$$

where the neutrino 4-momentum is $p^{\mu}=(E, \vec{p}), \omega_{k}=\sqrt{m^{2}+k^{2}}, \Omega_{k}=\sqrt{M^{2}+k^{2}}$ and the logarithmic functions [9, 110 are

$$
\begin{aligned}
L_{1}^{ \pm}(k)= & \ln \left(\frac{m^{2}-M^{2}+E^{2}-|\vec{p}|^{2}-2 E \omega_{k}-2 k|\vec{p}|}{m^{2}-M^{2}+E^{2}-|\vec{p}|^{2}-2 E \omega_{k}+2 k|\vec{p}|}\right) \\
& \pm \ln \left(\frac{m^{2}-M^{2}+E^{2}-|\vec{p}|^{2}+2 E \omega_{k}-2 k|\vec{p}|}{m^{2}-M^{2}+E^{2}-|\vec{p}|^{2}+2 E \omega_{k}+2 k|\vec{p}|}\right) \\
L_{2}^{ \pm}(k)= & \ln \left(\frac{M^{2}-m^{2}+E^{2}-|\vec{p}|^{2}+2 E \Omega_{k}+2 k|\vec{p}|}{M^{2}-m^{2}+E^{2}-|\vec{p}|^{2}+2 E \Omega_{k}-2 k|\vec{p}|}\right) \\
& \pm \ln \left(\frac{M^{2}-m^{2}+E^{2}-|\vec{p}|^{2}-2 E \Omega_{k}+2 k|\vec{p}|}{M^{2}-m^{2}+E^{2}-|\vec{p}|^{2}-2 E \Omega_{k}-2 k|\vec{p}|}\right) .
\end{aligned}
$$


$\Sigma^{F}$ represents the contribution of thermal fermions (electrons) and $\Sigma^{B}$ the contribution of thermal bosons, which are relevant for $T \sim M$. Notice that the term proportional to $e B \sigma_{3} \not p_{\|}$in Eq. (4.2) can be neglected because, for Dirac neutrinos on the mass-shell, $\sigma_{3} \not \not_{\|}=\frac{1}{2} \not \sigma_{3}=0$.

We consider now temperatures $T \ll M$. In this case the thermal bosons do not contribute, and we only need to consider the vacuum part of the $W$-propagator. For temperatures much lower than the $W$-mass, we can take the vacuum $W$-propagator in the $\xi=1$ gauge to be

$$
G_{0}^{\mu \nu}\left(x^{\prime}, x^{\prime \prime}\right) \simeq \phi\left(x^{\prime}, x^{\prime \prime}\right) \int \frac{d^{4} k}{(2 \pi)^{4}} e^{i k \cdot\left(x^{\prime}-x^{\prime \prime}\right)} \frac{g^{\mu \nu}}{M^{2}}\left(1-\frac{k^{2}}{M^{2}}\right)+O\left(1 / M^{6}\right) .
$$

The local piece does not contribute in a charge-symmetric medium, thus we can write the self-energy in a magnetic field to order $1 / M^{4}$ as

$$
\begin{aligned}
\Sigma= & -\frac{g^{2}}{M^{4}} \int_{-\infty}^{+\infty} \frac{d k}{(2 \pi)^{4}} f_{F}(k) \int_{-\infty}^{+\infty} d s e^{-\left[i s\left(m^{2}-k^{2}\right)+|s| \epsilon\right]}\left(\frac{\pi}{i s}\right)^{3 / 2}(e B) \\
& \times \gamma_{R}\left[\left(i \sigma_{3}+\cot e B s\right)\left(2 \gamma^{0} E s k^{2}-i \gamma^{3} p^{3}\right)-i \frac{e B s}{\sin ^{2} e B s} \not p_{\perp}\right] \gamma_{L} .
\end{aligned}
$$

We now use the following identities

$$
\cot e B s=i \sum_{n=0}^{\infty} \sum_{\lambda= \pm 1} e^{-i e B s(2 n+1-\lambda)}, \frac{e B}{\sin ^{2} e B s}=-\sum_{n=0}^{\infty} \sum_{\lambda= \pm 1} e B(2 n+1-\lambda) e^{-i e B s(2 n+1-\lambda)},
$$

change the variable of the $k$-integration, and obtain

$$
\begin{aligned}
\Sigma= & -\frac{g^{2}}{M^{4}} \frac{e B}{2 \pi^{2}} \int_{0}^{\infty} d k_{z} \gamma_{R}\left[\frac{f_{F}\left(E_{0,1, k_{z}}\right)}{E_{0,1, k_{z}}}\left(\gamma^{0} \vec{p} \cdot \hat{B} k_{z}^{2}+\vec{\gamma} \cdot \hat{B} E E_{0,1, k_{z}}^{2}\right)\right. \\
& \left.-\sum_{n=0}^{\infty} \sum_{\lambda= \pm 1} \frac{f_{F}\left(E_{n, \lambda, k_{z}}\right)}{E_{n, \lambda, k_{z}}}\left(\gamma^{0} E E_{n, \lambda, k_{z}}^{2}+\vec{\gamma} \cdot \hat{B} \vec{p} \cdot \hat{B} k_{z}^{2}+\frac{e B}{2}(2 n+1-\lambda) \not p_{\perp}\right)\right] \gamma_{L},
\end{aligned}
$$

where $E$ and $\vec{p}$ are the neutrino energy and momentum respectively, $E_{n, \lambda, k_{z}}$ is the energy of a thermal electron in the $n$-th Landau level (2.27) and $E_{0,1, k_{z}}$ the energy of thermal electrons in the lowest Landau level. The expression of the self-energy we derived is valid for $T \ll M$ and exact to all orders in the magnetic field. It can be written in a more manifestly covariant way using $\not p_{\perp}=\not p+\gamma^{0} E-(\vec{\gamma} \cdot \hat{B})(\vec{p} \cdot \hat{B})$. 
In order to obtain the dispersion relation we need to add the contribution of the bubble diagram with a $Z$-boson. This diagram is independent of the magnetic field and has been computed several years ago [8]. In the $\xi=1$ gauge and in a $C P$-symmetric plasma it is

$$
\Sigma_{Z}=-\frac{7 \pi^{2} g^{2} T^{4}}{180 M^{4}}\left(\frac{M^{2}}{M_{Z}^{2}}\right) \gamma_{R}\left(\gamma^{0} E+\frac{1}{4} \not p\right) \gamma_{L}
$$

We now concentrate on the situation where $m \ll T \ll M$ and $B \leq T^{2} / e$. In the early universe these conditions are reasonable between the $Q C D$ phase transition and nucleosynthesis. For the temperature and magnetic field in this range we can perform the integration in Eq. (4.8) and obtain

$$
\begin{aligned}
\Sigma= & -\frac{g^{2}}{M^{4}} \gamma_{R}\left[\frac{7 \pi^{2}}{90} T^{4}\left(1+\frac{M^{2}}{2 M_{Z}^{2}}\right)\left(\gamma^{0} E+\frac{1}{4} \not p\right)-\frac{e T^{2}}{24}\left(\gamma^{0} \vec{p} \cdot \vec{B}+E \not B\right)\right. \\
& \left.-\frac{e^{2}}{24 \pi^{2}} \ln \left(\frac{m}{T}\right)\left(-2 B^{2} \gamma^{0} E+2 \vec{p} \cdot \vec{B} \not B-B^{2} \not \supset\right)\right] \gamma_{L} .
\end{aligned}
$$

The part independent of $B$ agrees with the results of Ref. [8] and the part linear in $B$ agrees with the result of Ref. [2]. We were also able to obtain the $B^{2}$ term which could be important because of its dependence on $\ln \left(\frac{m}{T}\right)$. The resulting dispersion relation is

$$
E=|\vec{p}|\left[1-\frac{7 \pi^{2}}{90} \frac{g^{2} T^{4}}{M^{4}}\left(1+\frac{M^{2}}{2 M_{Z}^{2}}\right)\right]+\frac{g^{2} T^{2}}{12 M^{4}} e \vec{B} \cdot \vec{p}+\frac{g^{2}(e B)^{2}}{12 \pi^{2} M^{4}}|\vec{p}|\left[1-(\hat{B} \cdot \hat{p})^{2}\right] \ln \left(\frac{T}{m}\right)
$$

where the first two terms reproduce the result of Ref. [2], and the last one is very significant for $e B \sim T^{2} \gg m^{2}$.

\section{CONCLUSIONS}

We have studied the self-energy and dispersion relation of massless Dirac neutrinos in the presence of a constant magnetic field in a plasma. We obtain the self-energy in the $\xi=1$ gauge exact to all orders in the magnetic field, for all values of temperature below the critical temperature $\left(T_{C}\right)$ of the Weinberg-Salam model and all values of electron chemical potential. We then consider a $C P$-asymmetric plasma at temperature $T \ll M$. We have shown that 
in the weak-field limit our result agrees with those in the literature [1,2] and then derive the self-energy and dispersion relation to all orders in the $B$-field for an electron neutrino in a nonrelativistic and nondegenerate electron plasma, obtaining the index of refraction and effective mass of neutrino. The effective mass $m_{\nu_{e}}=-b$ given in Eq. (3.9) is calculated from the one-loop diagram at finite temperature in a constant and homogeneous magnetic field to all orders in the magnetic field strength. Its actual magnitude in the limit $\mu_{B} B / T \rightarrow 0$ is similar to that of the effective potential energy $V_{C}=\sqrt{2} G_{F} n_{e}$ induced by the coherent scatterings of a electron neutrino off electrons. [7]

Finally, we consider a charge-symmetric plasma, and derive from our main result of Section II an expression for the self-energy in a weak-field limit valid for $0 \leq T \leq T_{C}$. This expression could be very useful for studying magnetic properties of neutrinos for $T \sim M$. We then obtain the self-energy to all orders in $B$ for $T \ll M$ and then derive a very simple formula for the self-energy for $m \ll T \ll M$ and $B \leq T^{2} / e$ that contains also the term quadratic in $B$, while the terms linear and independent of $B$ are shown to agree with results from the literature [2,8]. We also obtain the dispersion relation.

\section{ACKNOWLEDGMENTS}

We wish to thank Gordon Feldman for helpful discussions. A. Erdas wishes to thank the High Energy Theory Group of the Johns Hopkins University for the hospitality extended to him during his several visits. This work is supported in part by the Basic Science Research Institute Program, Ministry of Education, Korea, 1997, Project No. BSRI-97-2418 and in part by Soong Sil University(THL). 


\section{REFERENCES}

[1] J. C. D’Olivo, J. F. Nieves and P. B. Pal, Phys. Rev. D $\underline{40}, 3679$ (1989)

[2] P. Elmfors, D. Grasso and G. Raffelt, Nucl. Phys. B $\underline{479} 3$ (1996)

[3] J. Schwinger, Phys. Rev. $\underline{82}, 664$ (1951)

[4] W. Dittrich and M. Reuter, Effective Lagrangians in Quantum Electrodynamics, Lecture Notes in Physics, (Springer-Verlag, 1985)

[5] A. Erdas and G. Feldman, Nucl. Phys. B $\underline{343}, 597$ (1990)

[6] P. Elmfors, D. Persson and B. Skagerstam, Nucl. Phys. B 464, 153 (1996)

[7] L. Wolfenstein, Phys. Rev. D 17, 2369 (1978); H. A. Bethe, Phys. Rev. Lett. $\underline{56}, 1305$ (1986); P. B. Pal and T. N. Pham, Phys. Rev. D $\underline{40}, 259$ (1989)

[8] D. Notzold and G. Raffelt, Nucl. Phys. B $\underline{307}, 924$ (1988)

[9] H. A. Weldon, Phys. Rev. D $\underline{26}, 2789$ (1982)

[10] C. Quimbay and S. Vargas-Castrillon, Nucl. Phys. B ㄴ51, 265 (1995) 


\section{FIGURES}

FIG. 1. The 3 diagrams relevant to the one-loop self-energy calculation; (a) the tadpole, (b) the bubble diagram with $W$-boson and (c) the bubble diagram with scalar. 


\section{FIGURES}

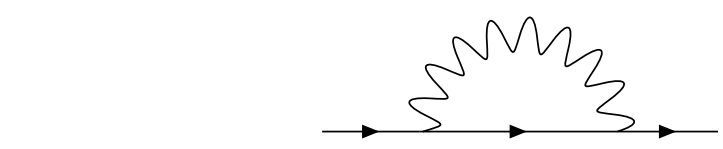

(a)

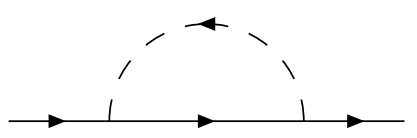

(b)

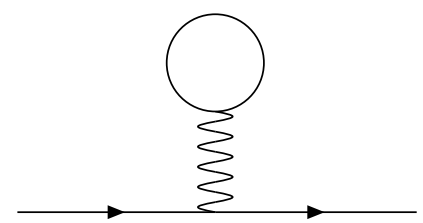

(c)

FIG. 1. The 3 diagrams relevant to the one-loop self-energy calculation; (a) the bubble diagram with $W$-boson, (b) the bubble diagram with scalar and (c) the tadpole. 